Revista Posgrado y Sociedad

Sistema de Estudios de Posgrado

Universidad Estatal a Distancia

ISSN 2215-2172

Costa Rica

revistaposgradoysoci@uned.ac.cr

\title{
La educación ante el enfoque sistémico
}

Education at the systemic approach

Nelson Zárate Arguedas

Saint Paul College

Alajuela, Costa Rica

Volumen 11, Número 2

Setiembre 2011

pp. $1-15$

Recibido: Julio, 2010

Aprobado: Julio, 2011 


\title{
Resumen
}

El artículo valora el enfoque sistémico dentro del quehacer científico y propositivo de la educación, tomando como referencia las diversas aportaciones de Mario Bunge y Fritjof Capra. En consecuencia, se plantean tópicos donde la educación se vincula con los sistemas sociales, económicos, políticos, culturales y ambientales para que, finalmente se focalice el papel de los estudios sociales dentro de la idea de sistema social mundial y el futuro humano.

Palabras clave: Educación; enfoque sistémico; sistemas sociales; sistemas económicos; sistemas culturales; estudios sociales; sistema social mundial

\begin{abstract}
The article evaluates the systemic approach in the scientific and proactive work of education, exploring the various contributions of Mario Bunge and Fritjof Capra. Therefore, topics are presented in which education is associated with social, economic, politic, cultural and environmental systems in order to ultimately bring the role of social studies into focus within the concept of the global social system and the future of humankind.
\end{abstract}

Keywords: Education; systemic approach; social systems; economic systems; cultural systems; social studies; global social system 


\section{Introducción}

Enseñar es facilitar el aprendizaje, estimular la creatividad, e infundir entusiasmo por la exploración del mundo y de las ideas"

(M. Bunge)

Las preguntas contrafactuales, por ser históricamente improcedentes, nos revelan por efecto contrario, que la realidad histórica y sus contextos se explican como procesos y relaciones sociales sustentados, reales y actuales. Proponer, por ejemplo, qué haría un hombre del medioevo con un ordenador, es contrafactual y es históricamente improcedente su planteamiento, sencillamente porque aquello no ha ocurrido (o no hay evidencia de que pudiese haber ocurrido).

La educación, de acuerdo con lo anterior, siendo una relación social, debe ser estudiada históricamente; es decir, de acuerdo con un devenir real, tanto para su comprensión como para las proposiciones que sobre ella se deban hacer a futuro.

Desde la perspectiva de Mario Bunge esto es cierto, siempre que dichas relaciones sociales sean entendidas dentro de la noción de sistema complejo, que admite una composición, un entorno y una estructura y que contempla con claridad los cuatro grandes subsistemas que amparan a todo sistema social; es decir: biológico, económico, político y cultural (1999 a, pp. 40 y 41).

Para Fritjof Capra, por su parte, el devenir de un sistema social debe ser entendido en el entorno de la significatividad que se deriva del vínculo entre la forma, la materia y el proceso (2003, p. 107).

Por eso, la educación para Capra se articula a partir de una amplia noción de cultura que permite la comunicación entre las personas, misma que ha cambiado también en función de la relación que establecen las sociedades con su tecnología, por ejemplo con la invención de la escritura o el uso de ordenadores en la actualidad. Ambos autores defienden el enfoque sistémico, pero no coinciden sobre ciertas apreciaciones filosóficas y epistemológicas ${ }^{1}$. Por eso, este artículo dará preferencia a la noción sistémica de Bunge, pero vinculando en lo posible las aportaciones de Capra ${ }^{2}$.

En las siguientes líneas se analizará la educación en sus retos presentes, procurando hacerlo dentro de un enfoque sistémico e histórico (es decir, vinculándolo con el supersistema social mundial) que contemple varios elementos relevantes como la sistematización de la cultura, el

\footnotetext{
${ }^{1}$ Por ejemplo Bunge se muestra crítico de la Escuela de Frankfurt, en particular de Habermas (1999, pp. 160 a 163); mientras Capra expone el pensamiento crítico y emancipador como un valioso e influyente aporte (2003, pp. 111 a 115).

2 La complejidad es sistémica (los sistemas sociales son complejos) y debe(n) ser entendida (dos) científicamente (matemáticamente); esa parece ser la mayor coincidencia entre Bunge y Capra. Capra subsume la biología, parte de ella para prolongar la comprensión sistémica ampliada. Bunge sostiene que cualquier subsistema debe ser visto con igual importancia e intensidad, lo importante es detectar, entender y relacionar los elementos que integran un sistema y su contexto.
} 
pensamiento convergente y el divergente (continuidad, cambio y adaptación), la innovación en la técnica, la tecnología y la ciencia; enfocando además la noción de tecnodemocracia dentro de criterios formativos. Todo ello con el objetivo de presentar posibles tópicos o elementos a considerar para las transformaciones del sistema educativo en América Latina, en el entendido de que la sociedad futura no puede planificarse considerando un solo aspecto programático, sino que debe integrar la economía, la política, la cultura, la biología, el ambiente y la educación, para la búsqueda de un modelo de sociedad no solo más justo y equilibrado, sino con más probabilidades de éxito (Bunge, 1999 b, 476 a 484).

\section{El sistema social mundial y la educación.}

Las políticas sociales, por ejemplo las relacionadas con la educación en América Latina, han sido implementadas muchas veces sin un respaldo diagnóstico confiable, ignorando las características y necesidades del sistema educativo $\mathrm{y}$ social. Se debe recordar que antes de proponer una solución es necesario entender el problema; y esa situación en apariencia obvia, ha sido desconocida tanto dentro de los procesos macro curriculares como los microcurriculares.

Las autoridades en educación implementan planes y programas que tienen poca significatividad en las comunidades y a la larga pasan a ser requerimientos que son resistidos o dejados de lado en el hecho educativo3.

Por su parte, el docente en ejercicio olvida $o$ no practica la evaluación diagnóstica constante y la atención multinivel, planeando $\mathrm{y}$ ejecutando lecciones que tienen un impacto muy diferente al esperado por él. Como se ve, el ejercicio diagnóstico debe ser siempre un primer paso en las aproximaciones sistémicas, tanto desde las perspectivas generales a las particulares y viceversa; incluyendo el estudio de la composición, entorno y estructura del sistema social que se pretenda entender 4 (Bunge, 1999 a, p.46).

Un primer paso para esta comprensión se asocia con el fundamento económico, que en nuestros días ha adquirido por efecto de la globalización, una connotación economicista.

\subsection{El economicismo dominante.}

Una referencia básica de la realidad del sistema social mundial en la actualidad, es que la economía de mercado, con fuerte influencia economicista, ha logrado imponer sus reglas al punto de influir poderosamente en las personas.

Dicha influencia economicista ha sido promovida desde los centros de poder (G-8, por ejemplo: Capra,2003,

\footnotetext{
3 Las reformas actúan verticalmente en virtud de la economía y la política. Para observar este asunto, véase a Solano (2001).

4 En el caso del presente artículo, solo se pretende establecer un punto de partida para una discusión sobre aspectos generales que se observan en el supersistema social mundial y que tienen incidencia en los procesos educativos y por lo tanto en posibles cambios dentro del sistema educativo.
} 
pp.171 a 186) y desde las corporaciones y empresas de gran capital. La respuesta que las personas han dado a esta tendencia, en muchos sentidos ha sido la búsqueda de una inclusión en términos de consumo, el cuál posibilita la satisfacción de necesidades más allá de las básicas, incursionando en aspectos simbólicos y de estatus que explican el éxito de los procesos de endeudamiento (tanto de las personas como de los Estados).

Para Bunge esto es un problema porque: "la ideología del mercado libre es moral y socialmente corrosiva al igual que cualquier otra forma de egoísmo. Es una suerte de anarquismo burgués." (1999 a, p. 45).

Uno de los impactos del economicismo dominante es que la exclusión social no se mira como un problema, siempre que exista una población suficientemente significativa para permitir el consumo que potencie el crecimiento económico.

Quien no sea capaz de consumir es porque no es competitivo (no se ha esforzado lo suficiente y obtiene lo que por equidad le corresponde). Esa desatención es lo que Bunge considera egoísta y corrosiva, porque en pos de poder consumir, las personas pueden optar por vías ilegales, violentas y sistemáticamente perniciosas, como el crimen organizado (incluido el de cuello blanco).

Así que una educación que responda servilmente al economicismo, promueve el egoísmo y atenta contra el equilibrio mismo del sistema social. En cambio, una educación que pueda promover la competencia tanto como la cooperación, sin que estos tengan que ser excluyentes, lograría que el sistema social no se desintegre y pueda encontrar relaciones humanas más justas y transformaciones sólidas, por un lado porque el sistema social no se estanca y por otro porque no se desequilibra5.

El economicismo no puede explicarlo todo y las relaciones humanas no se reducen a relaciones económicas; sin embargo, los ajustes necesarios para una implementación de políticas económicas inclusivas, deben proceder de decisiones y acciones políticas y de gobierno ${ }^{6}$.

Capra precisa que la transformación económica mundial de una economía mixta hacia la asunción neoliberal, se dio a partir del universo crítico que representó la estanflación. Una vez superado el problema inflacionario y de paralización productiva, el mundo corporativo tomó fuerza y logró la incorporación acelerada de procesos de globalización económica y cultural.

Dichos procesos para Capra son perniciosos por una gran cantidad de razones, dentro de las que sobresalen: la polarización de individuos y sociedades (exclusión), el automatismo

\footnotetext{
${ }^{5} \mathrm{Al}$ menos de acuerdo con el fundamento teórico sistémico de Bunge (1999 a, p.45).

$6 \mathrm{El}$ antropólogo García Canclini propone un accionar político que puede modificar las decisiones de Estados y Empresas a partir del consumo (o no consumo) coordinado de grupos humanos. Así, la comunidad de hispanos que voluntariamente dejaron de consumir unos días como protesta a ciertas leyes que consideraban perjudiciales para dicha comunidad, lograron pérdidas millonarias para el comercio y la inmediata acción correctiva de las autoridades e instituciones según su jurisprudencia. García Canclini sostiene que esta es una nueva forma de ejercer la ciudadanía (1995).
} 
del mercado (empoderamiento de lo virtual y separación entre el capital y la fuerza de trabajo tradicional) y el daño ecológico por causa del crecimiento económico insostenible (2003, pp. 179 a 194).

El mercado como componente eje de las relaciones humanas dentro de las circunstancias actuales, presenta por tanto dos relaciones principales que deben ser atendidas y en lo posible modificadas. La primera es la pretensión de que el desarrollo económico equivale a crecimiento económico (las personas intentan ser competitivas y eficientes dentro de los parámetros productivos de esta visión, la educación es una inversión para poder obtener empleo) (Bunge, $1999 \mathrm{~b}$, pp. 480 a 482); la segunda es que el objetivo fundamental de la vida humana es consumir todo lo que pueda (las corporaciones y empresas desarrollan distintas estrategias para conseguir la realización de sus mercancías, se valen de la condición mediática donde la masificación de la cultura influye en la cultura de masas, la educación promueve la competencia en su oferta académica y se convierte en un consumo más) (Capra, 2003, pp. 185 a 190).

Pero la economía se asocia con la política, porque las gestiones se derivan del ejercicio del poder y éste, es quien explica la naturaleza y dirección de los gobiernos y la gobernabilidad.

\subsection{Política, gobiernos y} gobernabilidad.

Para los países latinoamericanos, la tónica política en los últimos treinta años ha sido el Estado minimalista y neoliberal. Esto no es extraño por dos razones fundamentales: la primera es que las gestiones de política económica mundial utilizaron la condicionalidad cruzada7 para otorgar nuevos créditos a nuestros países, con tal de activar las economías y de esa manera recuperar la riqueza y posicionamiento estratégico.

La condicionalidad en si se ocupó de establecer reformas estructurales y apertura comercial. La segunda se relaciona con las transformaciones en el tipo y naturaleza de los gobiernos de la región, muchos de los cuales eran abiertamente totalitarios como en Chile, por gobiernos democráticos que pudiesen mediar en los procesos de pacificación de la región, los cuales eran indispensables para constituir redes comerciales más allá de las fronteras nacionales y los mercados internos.

$\mathrm{Si}$ bien, los procesos de pacificación y democratización fueron exitosos en casi todos los lugares, ello no supuso el fin de los conflictos sociales, ni mucho menos el logro de la paz al menos en un sentido civilista. Los gobiernos neoliberales elegidos democráticamente pronto encontraron resistencias y problemas de gobernabilidad.

La llegada en países como Brasil, Bolivia o Nicaragua de gobernantes de izquierda electos democráticamente, son un síntoma del descontento popular de los gobiernos neoliberales

\footnotetext{
7Hay que recordar que la condicionalidad cruzada es la forma en que los organismos económicos internacionales (Banco Mundial y FMI) se ponen de acuerdo para establecer las condiciones que deben asumir los países candidatos a un crédito. Generalmente dichas condiciones fueron en menoscabo de las políticas sociales en los años ochenta. Véase a Rodríguez-Ferrera (1997, pp. 45 a 46)
} 
de los noventa; o el éxito de iniciativas seudodemocráticas como la de Chaves en Venezuela. En síntesis, la experiencia política neoliberal ha polarizado las sociedades latinoamericanas, pero con un acomodo mayoritario en el grupo de los pobres.

Pero probablemente el factor más influyente en los modos que se ha organizado el poder en la región, corresponde a la influencia de los EE.UU. Ya sea por afiliación, por ejemplo con la firma de tratados comerciales o por la resistencia, como en el caso venezolano. $Y$ es que en este sentido, la globalización se muestra como un proceso que legitima la permanencia de la dominación imperialista (Quesada, 1998, pp. 23 a 40), con todas las implicaciones de fondo que ello contempla, para empezar la pérdida de autonomía.

Capra observa la cuestión política desde una óptica de la descomposición social, afirmando que la polarización social derivada de la globalización y el economicismo, ha incidido en el establecimiento de pugnas por control y mando en términos del pillaje y el crimen organizado (2003, pp. 194 a 199). Hay barrios en algunos países donde la policía se rehúsa a entrar y la vulnerabilidad del ciudadano común es cada vez mayor. Es decir, que en muchos sentidos, en América Latina se dificulta la condición de gobernabilidad.

La democracia latinoamericana ha operado con un criterio electoral válido (democracia representativa), pero la gestión democrática en si ha sido limitada (democracia participativa). En este sentido, la educación ha cumplido un papel reproductivo aunque de un impacto menor comparándolo con las plataformas mediáticas, incluyendo el creciente estimulo por el consumo.

De lo que se trata aquí, a nivel educativo es de promover una educación que promueva valores profundos de convivencia y ciudadanía, pero para que aquello tenga efecto, es necesaria una distribución más justa de la riqueza; que promueva el acceso al conocimiento, a la cultura sistematizada y a las soluciones sistémicas que requieren nuestros pueblos.

\subsection{Cultura sistematizada,}

\section{innovación y educación}

Dada la tremenda complejidad que implica razonar y analizar la cultura, en estas líneas se enfocará solamente la relación (cultura sistematizada)-(innovación-educación), considerando que un componente sustantivo en dicha relación es para Bunge la técnica (1999, pp.89 a 109) y para Capra la comunicación (significados) (2003, pp. 121 a 123).

Lo cierto es que en la actualidad, la educación tanto vista desde su connotación macro como micro, debe plantearse las relaciones educativas en virtud de dos necesidades imperiosas que requieren atención: a) la continuidad, que tiene que ver con el aprovechamiento del conocimiento científico, su comprensión y aplicación; y b) la innovación, que es lo que posibilita el cambio, la creatividad y debería ser el norte de toda investigación científica. Igual que se señaló con respecto a la competencia y cooperación; la continuidad y la 
innovación no deben ser ni excluyentes ni contrarias, sino complementos.

El problema para América Latina, es que la cuestión cultural en términos educativos y científicos es precaria. Por un lado, porque es imperativo para los centros económicos mundiales, que América Latina cumpla un rol de consumidor y no de productor; y por otro, porque las oligarquías locales han encontrado nichos muy cómodos al asociarse al gran capital transnacional y desatender la inversión en ciencia y tecnología; por ejemplo a través de las universidades o las propias iniciativas empresariales (Bunge, 1999 a, p. 134).

Pero aun cuando las universidades logren graduar científicos de alto nivel, esto no es garantía de que dichos graduados produzcan para el país o región, muchos emigran en lo que se conoce como fuga de cerebros ${ }^{8}$. Así que una acción correctiva en la formación de profesionales y científicos debe estar acompañada de un mejoramiento importante en las posibilidades de empleo y de salarios dignos; en especial para los educadores.

$\mathrm{Si}$ se quiere en verdad una sociedad competitiva y solidaria, entonces es menester un alto nivel educativo acompañado de un excelente desempeño en la investigación y la innovación. De igual manera, el ciudadano debe aspirar a la mejor educación posible para él y para la sociedad.

En cuanto a lo que significa el ejercicio docente, Capra permite una interesante aproximación a esa condición, que es técnica y relativa a la

\footnotetext{
${ }^{8}$ En realidad el caso de Frankling Chang y los graduados del Instituto tecnológico que trabajan para él en el proyecto del motor de plasma, es una excepción muy notable.
}

comunicación. Se trata de la enseñanza (y el aprendizaje) vista como proceso y como cuestión histórica (2003, pp. 117 a 130). En principio, la enseñanza universitaria en la actualidad combina los cuatro factores que interactuando hoy, han aparecido e influenciado a la construcción del pensamiento en formas e impactos diferentes a lo largo del devenir humano: a) la cultura oral, b) la cultura escrita quirográfica, c) la cultura escrita tipográfica y d) la cultura digital9.

En las sociedades sin escritura, la oralidad implicó el ritual, la repetición como formas de la tradición para la conservación del conocimiento y la cultura. A partir del descubrimiento de la escritura, diversos soportes (técnicas) permitieron la acumulación del conocimiento y la memoria histórica más allá de la capacidad de retención de una persona.

De las tablas de barro se pasó al papiro y de éste al pergamino, hasta el papel tal y como lo conocemos hoy. La escritura posibilitó otros procesos mentales, pero siempre dentro de un margen muy limitado de personas capaces de escribir y leer. Saber leer y escribir fue en extremo elitista, así como el manejo de ciertos niveles de la cultura sistematizada. Esa tendencia iba a cambiar un poco con la alfabetización griega, aunque el texto

\footnotetext{
9 El análisis histórico de estos factores de la comunicación y creación de la cultura sistematizada, merecerían muchas más páginas para su tratamiento, pero es imposible dentro de los límites de este trabajo. Así que solamente se planteará un muy breve esbozo de cada uno y en conjunto. Sin embargo, para una contrastación rápida es recomendable el texto de Avendaño (2005), en quien se basa el esquema de exposición de este asunto.
} 
(códice) por ser escrito a mano era difícil de producir y leer.

La copia de libros en el Medioevo estuvo a cargo de los monjes en las abadías y en las bibliotecas, pero el proceso era muy lento. Con la llegada de la xilografía y en especial de la imprenta de tipos móviles, la producción de libros se abarató y masificó, apareciendo también otros medios escritos muy influyentes como los periódicos. Ya en el siglo XX, el maquinismo había logrado la aparición de editoriales y ediciones de bolsillo que alcanzaron ediciones que se vendieron por millones.

El texto se convirtió en la referencia obligada de cualquier idea de educación. Los nacionalismos promovieron la educación con textos (libros de texto) escritos en lenguas vernáculas $\mathrm{y}$ esto no ocurrió sin resistencias.

Pero ha sido en los últimos cincuenta años, en que la forma de conservar y transmitir información y conocimientos ha sido revolucionaria en muchos sentidos: la aparición de la radio, la imagen en el cine y la televisión y la era de las redes informáticas, plantean una nueva condición en el ámbito educativo que debe ser tomado en cuenta: el soporte informático, que por ejemplo rompe con la idea lineal de un texto, para posibilitar lecturas que saltan de un texto a otro por medio de hipervínculos (se trata de hipertextos) que hacen que las lecturas y los conocimientos se adapten a las necesidades y decisiones de un lector en particular, en patrones diferentes a otro lector en las mismas condiciones.
La razón de haber realizado este esbozo, es que Capra invita a entender el aprendizaje humano dentro de un devenir histórico condicionado y modelado por la cultura, por medio de los códigos y sus significados. Es la palabra la que permite las ideas, y son los soportes y condiciones técnicas con que se relacionen las personas, los que permiten elaborar diferentes procesos mentales.

Cuando se piensa en la sistematización de la cultura hoy y su vínculo con la educación, resulta una compleja imbricación de soportes que actúan e interactúan sincrónicamente, ampliando en muchos sentidos las posibilidades de comunicación humana. Por consiguiente, el resultado cultural de ese devenir no se puede obviar en ninguna de sus manifestaciones.

La educación hoy debe entender y apropiarse de dichas manifestaciones culturales, puesto que al interactuar proponen formas de construcción del pensamiento inéditas, que deben ser estudiadas, con el fin de eludir sus limitaciones y potenciar sus fortalezas.

La civilización tecnológica, tal y como están las cosas es irreversible; debemos relacionarnos con las nuevas tecnologías con ventaja y esto es parte de lo que Capra llama "estructura semántica” (2003, p.108). Para Bunge, dicha relación debe ampararse en la tecnodemocracia que puede interpretarse como una forma de maximizar los beneficios de la tecnología en el mayor número de miembros de la sociedad y minimizar sus efectos maléficos (1999, p. 105), esto gracias al ejercicio ciudadano responsable, consciente y democrático. 
Tal ejercicio, tiene mucho que ver con otro elemento de importancia: la vida misma, el ser humano en relación con el sistema natural en pleno.

\subsection{La humanidad, el medio ambiente y la vida.}

Fue Alvin Toffler (1973, p. 6) quien difundió la idea de que desde la aparición del CroMagnon, harán unos cincuenta mil años atrás hasta el presente, han pasado por esta tierra poco más de ochocientas generaciones ${ }^{10}$ de homosapiens. Pero es la última generación la que produjo la mayor cantidad de innovaciones tecnológicas y producción de bienes y servicios.

La única que ha tenido la capacidad real de acabar con el planeta con el simple arte de apretar un botón.

El efecto primero de esta idea, es que el conocimiento y su aplicación acarrean responsabilidades. Como se vio en el apartado anterior, la técnica no es neutral y ha tendido a ser excluyente; si a eso le sumamos el andamiaje economicista que ampara a dicha técnica, el resultado es una "racionalidad de la eficiencia y la rentabilidad" que es, dentro del pensamiento de Bunge y el mismo Capra: irracional. Es decir, admitir que el sistema social mundial está inserto en un sistema natural global, es una cuestión evidente; pero el mercado ha legitimado a la eficiencia aun con el costo que puede representar el agotamiento de los recursos que nos permiten estar vivos.

\footnotetext{
${ }^{10}$ Toffler dice ampararse en el criterio de generación que utilizó Ortega y Gasset, por períodos de sesenta años.
}

De acuerdo con Bunge el sistema social mundial no es un ser vivo (1999 a, p.30), pero los elementos humanos que lo componen sí lo están; en tanto Capra, intenta visualizarlo como un sistema vivo o vivo en grado variable (2003, p. 117).

De cualquier manera, lo importante es que la vida es una cuestión prioritaria en ambos discursos; por consiguiente, una racionalidad de la eficiencia que no admita la vida, o la destruya sin empacho es suicida. Si la eficiencia fuera cortar la rama de un árbol, ¿a quién aplaudiría el economicismo? Pues al primero en cortar una rama. No importa si es la rama en la que se está sentado ${ }^{11}$.

La educación que se ocupe de crear una conciencia a favor de la vida, debe hacerlo con base en razones (científicas) y no como el resultado panfletario del miedo y la manipulación. El conservacionismo iluso, no debe impedir el mejoramiento en la calidad de vida en las personas y las sociedades; lo mismo que el discurso ecológico no debe convertirse en un estilo de consumo más, en favor de corporaciones emergentes e igualmente tendenciosas.

Parece que nuevamente resulta importante la sugerencia de Bunge en el sentido de promover la participación democrática ciudadana en estos menesteres. Se trata, en última instancia, de una educación y de un proyecto de sociedad que promueva la vida con criterios racionales $\mathrm{y}$ equilibrados y altamente participativos, inclusivos y comprometidos.

\footnotetext{
${ }^{11}$ El ejemplo es de Hinkelammert (1995, pp. 137 a 145)
} 
La necesidad de fortalecer el ámbito de la andragogía.

Piaget no estudió la epistemología en el adulto, aunque muchos de sus parámetros de análisis formalizantes tenían como norte la consideración de que el pensamiento adulto era equivalente al pensamiento formal, y esta es una perspectiva claramente incompleta sobre la que se hace necesario un mayor despliegue investigativo, tanto en materia de la psicología humana, como en lo referido a la neurología y en general la biología.

Los procesos madurativos no acaban en la adolescencia, las transformaciones ontogenéticas continúan a lo largo de toda la vida humana y los condicionamientos epigenéticos jamás cesan. Significa que el cerebro cambia, sigue cambiando y especializándose; tiene plasticidad por lo que es posible mejorar las condiciones de desempeño de los niveles mentales superiores (Restak, 2005, pp. 169 a 207).

Pero también la psicología en las personas evoluciona, por ejemplo si se consideran ciertas tareas y condiciones en la vida: las personas entre los 17 y 22 años hacen compromisos tentativos y están en la búsqueda de una independencia propia de la adultez joven. De los 22 a 29 se ocupan de construir una estructura de vida, de tener una carrera universitaria o una forma de vida, un proyecto de vida. De los 29 a los 33 ocurre una reevaluación de la etapa anterior, un reposicionamiento. De los 33 a los 40 se crea una segunda estructura de vida, sobre la base de la experiencia y la independencia.
A partir de los cuarenta se formulan preguntas sobre el qué se ha hecho con la vida, es una revisión autocrítica profunda que lleva a plantearse nuevas estructuras de vida hasta los cincuenta años. De los cincuenta en adelante la revisión y replanteamiento es constante, pero al mismo tiempo se goza de mayor equilibrio emocional para pasar a la etapa final que compete al retiro y a la asimilación de la frontera de la vejez, que en nuestro contexto se razona después de los 65 años (Knowles, 2006, p. 195).

Independientemente que estemos de acuerdo o no con la anterior esquematización de la construcción evolutiva, lo cierto es que las personas estamos permanentemente expuestas al cambio y a la transformación en la que siguen interactuando, tal y como la psicogenética y la neurogenética lo han evidenciado, la inteligencia (intelectualidad) y la afectividad (emociones).

Por lo tanto, es pertinente $\mathrm{y}$ necesario que se amplíen las investigaciones en materia de la andragogía en toda su complejidad y alcance, debido a que la ecuación y el aprendizaje deben ser entendidos como condiciones permanentes en el ser humano.

\section{La educación, futuro y cultura de la esperanza.}

Hasta este punto, lo que queda claro es que el devenir histórico del sistema social mundial y la educación dentro de él, tienen una complejidad multivectorial. La escurridiza noción de 
presente, constantemente evoca al futuro y el futuro es una abstracción, una prefiguración que corresponde al mundo de lo posible.

Dicha prefiguración, de acuerdo con los señalamientos ya hechos, debería ampararse en el conocimiento del pasado, en la ciencia y la razón que permita planificar ese futuro. La educación debería ser un medio para la constitución misma del conocimiento (convergente) para la implementación de las soluciones que requiere el futuro (conocimiento divergente y prognosis).

De acuerdo con los autores que amparan este artículo, tal proceso tiene sentido dentro de un pensamiento sistémico de la sociedad; pero además, la atención y demarcación de ese futuro es imperativo, urgente; porque se cierne en la humanidad una propensión a las más diversas amenazas, incluyendo su propia destrucción (Bunge, 1999 b, pp. 472 a 474).

$\mathrm{Al}$ parecer, entre más compleja y sistematizada se ha vuelto la humanidad, mayor ha sido el impacto sobre el medio. La tecnología, el crecimiento económico y el consumismo, son parte de esos factores de impacto. Por su parte Capra plantea el problema de las redes del capitalismo global (2003, p. 171 en adelante), la sociedad de la información y las nuevas maneras en que se están relacionando las personas, fortaleciendo la lógica capitalista que supone el consumo.

El hecho de poder entender que existen muchos y complicados problemas del presente y que se proyectan a futuro, implica una responsabilidad para quienes formamos parte de esa conciencia. Por un lado porque, como apunta Capra, la gestión de la globalización no es casual, sino que ha sido diseñada por el mundo corporativo y las organizaciones económicas internacionales (2003, p. 182) y por otro lado, ese poder trasciende la capacidad de respuesta de los gobiernos y estados en particular, para lo cual es necesario desarrollar estrategias que integren a las comunidades locales con las comunidades internacionales, en pos de una causa común que es la búsqueda de un mejor futuro para las mayorías (en lo posible para todos).

La educación debe promover el pensamiento creativo, porque en él se encuentran las alternativas, los sueños o utopías que eventualmente podrían hacerse realidad. Lo que esto significa es que los sueños en si mismos no son suficientes: se requiere de transformar la sociedad (Bunge lo llama ingeniería social, 1999, p. 476).

Por su parte, Capra apunta a que las alternativas surgen de proposiciones viables dentro de un andamiaje ecológico y sostenible, que tiene implicaciones educativas en término de divulgación y mejoramiento. Ejemplo de lo anterior es lo que este autor llama: ecoalfabetización y ecodiseño (2003, pp. 290 a 325).

De acuerdo con Bunge, la mejor manera de promover una ingeniería social, es mediante el establecimiento de una reforma sistémica, planificada y democrática (1999 a, 476 a 480). Si se toma por caso una intención desarrollista, ésta no debe ser unidimensional, por ejemplo, centrando todas las expectativas en el crecimiento económico o acaso el ecologismo. La política debe ser integral (sistémica) y multidimensional.

El desarrollo por tanto, tiene distintos indicadores que competen a la obtención de un alto nivel en la calidad 
de vida de las personas y las sociedades en el ámbito ambiental, biológico, económico, político y cultural. Esto significa que la planificación del cambio social, debe ser el resultado de un consenso social, pero orientado por la labor coordinada por especialistas de forma inter y transdisciplinaria.

\subsection{La Educación en los años por venir.}

En relación con las condiciones actuales del desarrollo científico y tecnológico, uno de los puntos de partida de cualquier política educativa, aparte del indispensable diagnóstico sistémico, es el reconocimiento de la aceleración del cambio en términos del conocimiento y la técnica. La planificación, debe contemplar mecanismos y formas de adaptación y actualización constantes, porque de otro modo, la escuela se rezaga respecto a la realidad histórica (Bunge, 1999 a, p.136).

La enseñanza debe facilitar el aprendizaje y estimular la creatividad y la educación en pleno, debe ser una oportunidad para promover el conocimiento científico y el bienestar humano.

$\mathrm{Si}$ en realidad se quiere un desarrollo integral y se entiende que una educación de altísima calidad es necesaria para ese fin, entonces se requiere que las mejores mentes y vocaciones, estén a cargo de esta labor. Esto no es posible con condiciones materiales precarias para los maestros; hay que hacer atractiva la labor docente por medio de la compensación salarial y otros estímulos edificantes (Bunge, 1999 a, p. 137).
Otras consideraciones válidas se relacionan con la distribución de horas y énfasis de la enseñanza, investigación y reforma de los métodos de enseñanza, por ejemplo, maximizando los trabajos prácticos y los espacios de proyección social.

En este sentido, la educación debe orientar una formación integral y ética que sirva de fundamento al ejercicio político de los gobernantes y de una ciudadanía comprometida.

\subsection{La educación respecto a los estudios sociales.}

Para Bunge, la posibilidad de que la educación cumpla un papel protagónico y acertado en la construcción de la humanidad del futuro, depende de cuán próxima esté la misma del pensamiento ético, científico y técnico. Si por el contrario prevalece lo que este autor llama oscurantismo (en especial de los estudios sociales contemporáneos), el resultado sería el error y la confusión (1999 a, pp. 151 a 152).

Bunge se muestra crítico y escéptico del pensamiento que no se base en las ciencias, que para él son metódicas y matemáticas. La sociología fenomenológica, la etnometodología y la hermeneútica, entre otros elementos, son parte de lo que Bunge llama olas románticas y contrailustradas o contaminadas (1999 a, pp. 154 a 170).

Bunge sostiene que es necesario el conocimiento social más veraz y profundo posible y sostiene que muchos de los límites que se les ha asignado a las ciencias sociales, son falaces (1999 b, pp. 484 a 486). Si bien las ciencias sociales aun no alcanzan el 
nivel y confiabilidad de las ciencias naturales, podrían hacerlo bajo ciertas condiciones, como por ejemplo la adopción del sistemismo ontológico, el sistemismo metodológico, el realismo científico y la crítica al irracionalismo, el respeto a las normas científicas y el conocimiento y planteamiento de una filosofía de las ciencias sociales que sea sólida (1999 b,p. 448).

\subsection{Hacia una cultura de la esperanza.}

Tanto Capra como Bunge, después de su esfuerzo crítico, toman posturas humanistas y esperanzadoras. Eso debe hacer también la educación desde todos los niveles y perspectivas; es decir, relacionarse con la información para que se convierta en conocimiento, permitir que ese conocimiento sea crítico, creativo, innovador y bien fundamentado, para que el cambio social se base en ideas y no en fuerzas como el miedo, que tanto daño pueden causar.

Un primer paso para vislumbrar la esperanza es el logro de la inclusión y la justicia social.

\section{Conclusiones}

La educación debe ser entendida de modo sistémico e histórico, para poder comprender los problemas, las limitaciones y necesidades que ésta pueda presentar; con lo cual las reformas y estrategias educativas puedan responder acertadamente y en proximidad a criterios científicos.
En la actualidad, las condiciones económicas tienden a un economicismo que es pernicioso y que afecta a la educación de muchas maneras. Por lo tanto, se debe implementar criterios económicos que promuevan el equilibrio y la justicia social.

Es importante que los gobernantes latinoamericanos actuales, propongan estas políticas económicas más justas, para de ese modo, enfrentar los serios problemas de polarización y exclusión social que incrementan la violencia y el crimen.

La educación puede cumplir un papel protagónico en el cambio social, pero debe promover para ello un mejor manejo y conocimiento de las "estructuras semióticas", los soportes y el entorno técnico en la era de la información y el conocimiento; para poder relacionarse de un modo responsable con la vida y la naturaleza.

Todo esto requiere de acciones conjuntas, de distintos sectores de la sociedad (tecnodemocracia) para poder diseñar un cambio social basado en el enfoque sistémico, que promueva una noción integral y multidireccional del desarrollo y que no pierda de vista la importancia del sistema natural en el que vivimos.

Pero la educación debe promover conocimientos veraces y éticos, para lo cual se debe promover la investigación y divulgación científica, en especial en los estudios sociales. El objetivo final es desarrollar una humanidad mejor, con base en una cultura de la esperanza y no del miedo o el engaño. 


\section{Nelson Zárate-Arguedas}

\section{Referencias}

Avendaño, F. (2005). La cultura escrita ya no es lo que era. Argentina: HomoSapiens.

Capra, F. (2003) Las conexiones ocultas. Barcelona: Ed. Anagrama.

Bunge, M. (1999 a) Sistemas Sociales y su filosofía. Buenos Aires: Editorial suramericana.

Bunge, M. (1999 b) Las Ciencias Sociales en Discusión: Una perspectiva filosófica. Buenos Aires: Editorial Sudamericana.

García, N. (1995) Consumidores y ciudadanos. Conflictos multiculturales de la globalización. México: Grijalbo.

Hinkelammert, F. (1995). Cultura de la esperanza y sociedad sin exclusión. San José: DEI.

Quesada, R. (1998). Globalización y deshumanización. Dos caras del capitalismo avanzado. Heredia: EUNA.

Rodriguez-Ferrea, J. (1997). La economía mundial y el desarrollo. Madrid: Acento.

Solano, J. (2001) Educación y Desarrollo en América Latina: Un análisis histórico conceptual. Heredia: EUNA.

Toffler, A. (1973). El "shock" del futuro. Barcelona: Plaza \& Janes.

\section{Nota acerca del autor}

\section{Nelson Zárate Arguedas}

Con formación inicial como historiador y profesor de Estudios Sociales. Licenciado en la enseñanza de los Estudios Sociales (UNA. 1998). Máster en Psicopedagogía (UNED, 2007). Actualmente trabaja en el Saint Paul College y es doctorando del Doctorado Latinoamericano de Educación de la UNED. Fue docente en la Universidad Nacional 2001-2008. Es autor de diversos artículos publicados en Revistas como "Perspectivas" (UNA) y "Posgrado y Sociedad" (UNED)

Correo electrónico: nejorzar@racsa.co.cr 